University of New Hampshire

University of New Hampshire Scholars' Repository

Space Science Center

Institute for the Study of Earth, Oceans, and

Space (EOS)

2000

\title{
COMPTEL time-averaged all-sky point source analysis
}

W Collmar

Max-Planck-Institut für extraterrestriche Physik

V Schonfelder

Max-Planck-Institut für extraterrestrische Physik

A W. Strong

Max-Planck-Institut für extraterrestriche Physik

H Bloemen

Space Research Organization of the Netherlands

W Hermsen

Space Research Organization of the Netherlands (SRON)

See next page for additional authors

Follow this and additional works at: https://scholars.unh.edu/ssc

Part of the Astrophysics and Astronomy Commons

\section{Recommended Citation}

COMPTEL time-averaged all-sky point source analysis Collmar, W. and Schönfelder, V. and Strong, A. W. and Bloemen, $\mathrm{H}$. and Hermsen, W. and McConnell, M. and Ryan, J. and Bennett, K., AIP Conference Proceedings, 510, 591-595 (2000), DOl:http://dx.doi.org/10.1063/1.1303271

This Conference Proceeding is brought to you for free and open access by the Institute for the Study of Earth, Oceans, and Space (EOS) at University of New Hampshire Scholars' Repository. It has been accepted for inclusion in Space Science Center by an authorized administrator of University of New Hampshire Scholars' Repository. For more information, please contact Scholarly.Communication@unh.edu. 


\section{Authors}

W Collmar, V Schonfelder, A W. Strong, H Bloemen, W Hermsen, Mark L. McConnell, James M. Ryan, and K Bennett 


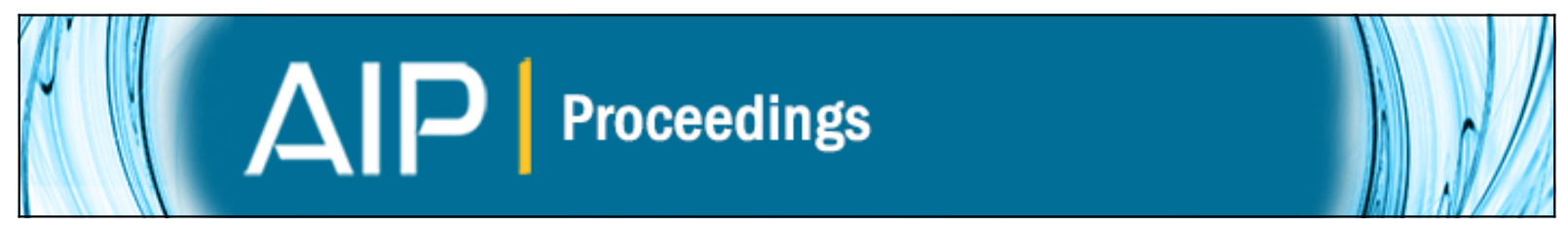

\section{COMPTEL time-averaged all-sky point source analysis}

W. Collmar, V. Schönfelder, A. W. Strong, H. Bloemen, W. Hermsen, M. McConnell, J. Ryan, and K. Bennett

Citation: AIP Conference Proceedings 510, 591 (2000); doi: 10.1063/1.1303271

View online: http://dx.doi.org/10.1063/1.1303271

View Table of Contents: http://scitation.aip.org/content/aip/proceeding/aipcp/510?ver=pdfcov

Published by the AIP Publishing

\section{Articles you may be interested in}

The spectral properties of the GRB prompt gammaray emission observed by the Suzaku Wideband Allsky Monitor

AIP Conf. Proc. 1000, 101 (2008); 10.1063/1.2943418

AGILE sky exposure and sensitivity maps

AIP Conf. Proc. 587, 759 (2001); 10.1063/1.1419495

All sky monitor for energetic $\mathrm{Y}$-rays

AIP Conf. Proc. 558, 594 (2001); 10.1063/1.1370831

Preliminary results from a new analysis method for EGRET data

AIP Conf. Proc. 510, 606 (2000); 10.1063/1.1303274

Improved COMPTEL maps of the milky way

AIP Conf. Proc. 510, 586 (2000); 10.1063/1.1303270 


\title{
COMPTEL Time-Averaged All-Sky Point Source Analysis
}

\author{
W. Collmar*, V. Schönfelder*, A.W. Strong*, H. Bloemen ${ }^{+}$, \\ W. Hermsen ${ }^{+}$, M. McConnell ${ }^{\dagger}, J_{\text {. Ryan }}^{\dagger}$, K. Bennett ${ }^{@}$ \\ * Max-Planck-Institut für extraterrestrische Physik, Postfach 1603, 85740 Garching, Germany \\ +SRON-Utrecht, Sorbonnelaan 2, NL-3584 CA Utrecht, The Netherlands \\ † Universtity of New Hampshire, Durham NH 03824-3525, USA \\ @ Astrophysics Division, ESTEC, NL-2200 AG Noordwijk, The Netherlands
}

\begin{abstract}
We use all COMPTEL data from the beginning of the CGRO mission (April '91) up to the end of CGRO Cycle 6 (November '97) to carry out all-sky point source analyses in the four standard COMPTEL energy bands for different time periods. We apply our standard maximum-likelihood method to generate all-sky significance and flux maps for point sources by subtracting off the diffuse emission components via model fitting. In addition, fluxes of known sources have been determined for individual CGRO Phases/Cycles to generate lightcurves with a time resolution of the order of one year. The goal of the analysis is to derive quantitative results - significances, fluxes, light curves - of our brightest and most significant sources such as $3 \mathrm{C} 273$, and to search for additional new COMPTEL sources, showing up in time-averaged maps only.
\end{abstract}

\section{INTRODUCTION}

The imaging COMPTEL experiment aboard CGRO is the pioneering satellite experiment of the MeV-sky $(\sim 1-30 \mathrm{MeV})$. For a detailed description of COMPTEL see [1]. One of COMPTEL's prime goals is the generation of all-sky maps, which provide a summary on the $\mathrm{MeV}$-sky in total. This goal has been achieved by e.g. [2], [3] who generated maximum-entropy all-sky images and by [4], who generated the first COMPTEL all-sky maximum-likelihood maps, which - compared to maximum-entropy ones - have the advantage of providing quantitative results like significances and fluxes of source features. Here we present all-sky maximumlikelihood maps from which models of the diffuse emission have been removed. Our emphasis is on AGN. For a discussion on the method see [5] in these proceedings.

The main analysis goals are 1) to derive a summary of known COMPTEL point sources, 2) to search for further point sources, 3) to derive time-averaged quantitative parameters ('first order') of our brightest point sources, i.e., significances, fluxes, MeV-spectra, and possible time variability, and 4) to further investigate our data and analysis methods.

CP510, The Fifth Compton Symposium, edited by M. L. McConnell and J. M. Ryan (c) 2000 American Institute of Physics 1-56396-932-7/00/\$17.00 


\section{DATA AND ANALYSIS METHOD}

Using all data from the beginning of the CGRO mission (April '91) to the end of CGRO Cycle VI (Nov. '97), we generated a consistent database of relevant COMPTEL data sets (events, exposure, geometry) for individual CGRO viewing periods (VPs) in the 4 standard energy bands $(0.75-1,1-3,3-10,10-30 \mathrm{MeV})$ in galactic coordinates by applying consistent data selections. This database was supplemented by relevant data sets containing models describing the galactic diffuse $\gamma$-ray emission (HI, CO, and inverse-Compton components) and the isotropic extragalactic $\gamma$-ray background emission. To check for time variability of $\gamma$-ray sources these data sets were combined for different time periods: the six individual CGRO Phases/Cycles, the sum of all data (CGRO Phases I-VI; April '91 - Nov. '97) as well as the first (CGRO Phases I-III; April '91 - Oct. '94) and the second half (CGRO Phases IV-VI; Oct. '94 - Nov. '97). Each set of all-sky data is analysed by our standard maximum-likelihood method which simultaneously 'handles' individual VPs, generates, iteratively, a background model (see [6]), and finally generates significance and flux maps and/or significances and fluxes for individual sources. Because we are interested in point sources, the diffuse emission is always removed in the fitting procedure (e.g. Figure 1). For !he derivation of the source fluxes (see Figure 2 as an example), the point sources of interest (e.g. 3C 273, Cyg X-1) have additionally been included in the fitting procedure. We like to mention however, that the results derived by such all-sky fits should be considered correct to first order only. To derive final/optimal results for a particular source, a dedicated analysis has to be carried out, which e.g. makes several cross checks by applying different background models and would take into account the presence of other source features in the region of interest. Also, along the galactic plane the results depend on the 'goodness' of the applied diffuse emission models for the MeV-band.

\section{RESULTS}

The significance maps in Figure 1, which contain all data of the first 6.5 years of the COMPTEL mission, are the first COMPTEL all-sky point source maps in the continuum bands. They provide a summary of the on-average brightest and most significant MeV-sources. Similar mape focussing on the Galactic plane only are given elsewhere in these proceedings ([5]). The Crab - for display reasons removed in all maps of Figure 1 - is by far the most significant COMPTEL point source. In the 1-3 MeV band for example it reaches a significance of $\sim 110 \sigma$ (i.e. a likelihood ratio of $\sim 12000$ ) for the CGRO Phase I-VI period. With significances of $\sim 11 \sigma, \sim 10 \sigma$, and $\sim 6 \sigma$ in the 1-3, 3-10, and $10-30 \mathrm{MeV}$ bands is the quasar 3C 273 found to be on average the second most significant point source. Its fluxes in these bands are between $10 \%$ and $15 \%$ of the Crab flux. Several other extragalactic (e.g. 3C 279, PKS 0528+134, Cen A) and galactic (e.g. Cyg X-1, PSR 1509-58, a known but unidentified source at $\left.1 ; b: 18^{\circ} ; 0^{\circ}\right)$ sources are visible as well. In addition there 

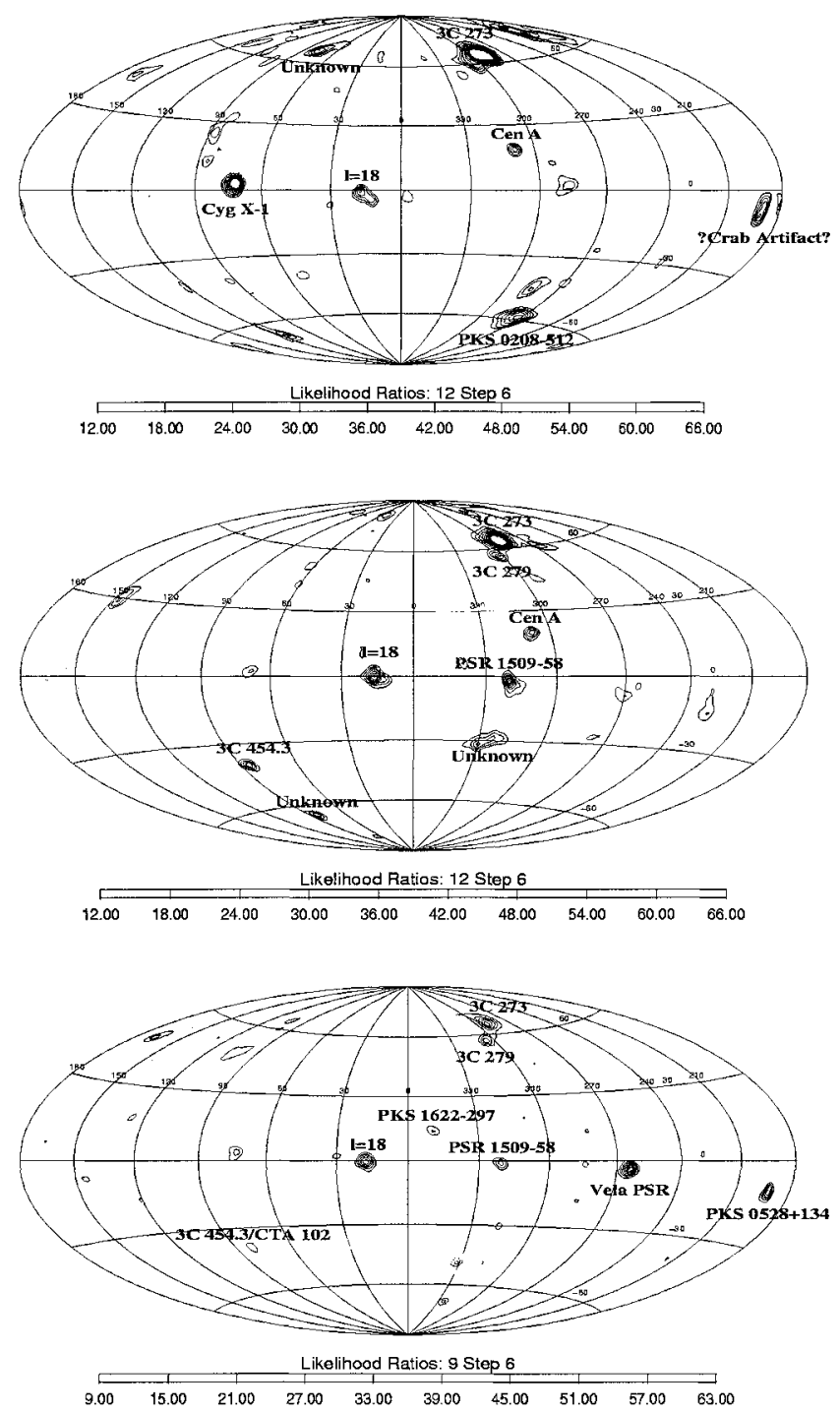

FIGURE 1. COMPTEL time-averaged maximum-likelihood point source all-sky maps in the 1-3 (upper), 3-10 (middle), and 10-30 MeV (lower panel) energy bands for the time period April '91 to November ' 97 , i.e. CGRO phases I-VI. The galactic and extragalactic diffuse emission as well as the emission from the Crab have been subtracted off via model fitting. For the 1-3 MeV and the $3-10 \mathrm{MeV}$ significance maps the contour lines start at a likelihood ratio value of $12(\sim 3.5 \sigma$ for a known source; $\chi_{1}^{2}$-statistics) and for the $10-30 \mathrm{MeV}$ map at a likelihood ratio value of 9 (3.0 $\sigma$ for a known source) with steps of 6 for all maps. The most significant source features are labeled. 

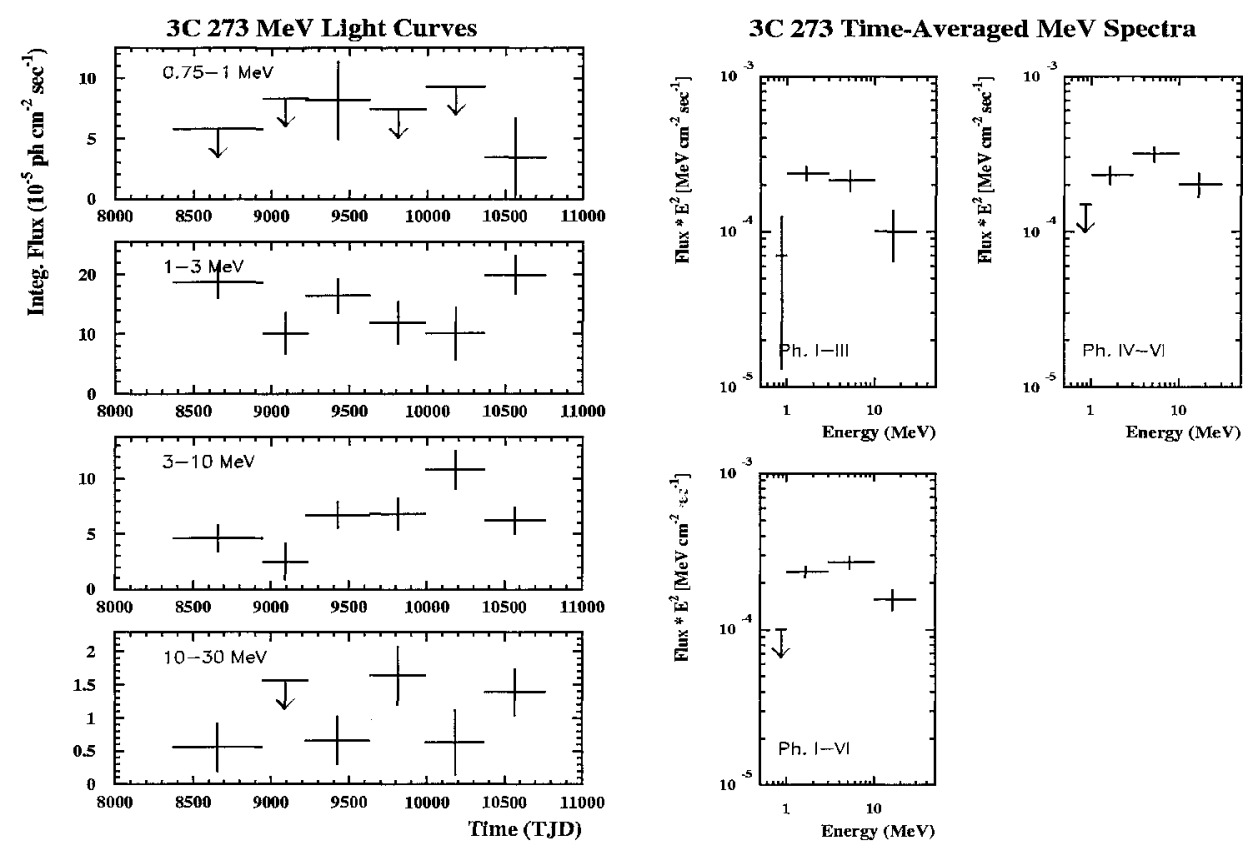

FIGURE 2. Left: COMPTEL light curves of the quasar $3 \mathrm{C} 273$ in the 4 standard bands as derived from all-sky flux fitting. Each flux point is averaged over an individual CGRO phase. The error bars are $1 \sigma$ and the upper limits are $2 \sigma$.

Right: COMPTEL MeV spectra of 3C 273 for the sum of all CGRO phase I-III (April '91 - Oct. '94), IV-VI (Oct. '94 - Nov. '97), and I-VI (April '91 - Nov. '97) data as derived from all-sky flux fitting. The error bars are $1 \sigma$ and the upper limits are $2 \sigma$.

are indications for previously unknown source features like at $1 ; \mathrm{b}: 75^{\circ} ;+65^{\circ}$ in the 1-3 MeV map and at 1;b: $85^{\circ} ;-65^{\circ}$ in the $3-10 \mathrm{MeV}$ map for example. Such spots are promising candidates for further dedicated analyses. This time-averaged approach suppresses sources which flare up only in short time periods. Therefore the maps show fewer sources than are listed in the COMPTEL source catalog (see [7]).

For all bright and significant COMPTEL sources we have derived fluxes in our 4 standard energy bands for the different time periods mentioned above, and have combined them to $\mathrm{MeV}$ light curves and spectra. Some results for $3 \mathrm{C} 273$ are shown as an example in Figure 2. In the 1-10 MeV energy band 3C 273 is detected in each CGRO Phase/Cycle i.e. in time periods of typically 1 year. The flux turns out to be rather stable and varies only within a factor of $\sim 2$ in the $1-3 \mathrm{MeV}$ and within a factor of $\sim 4$ in the $3-10 \mathrm{MeV}$ energy band. The spectra show the same trend. Whereas the flux below $3 \mathrm{MeV}$ turns out to be same for both halves, there is an indication that at the upper COMPTEL energies $(>3 \mathrm{MeV})$ the source was brighter during the second half. All three spectra clearly show the spectral turnover 
TABLE 1. Detection significances $(\sigma)$ in 3 different energy bands of some known COMPTEL AGN sources (plus Crab) as derived by this all-sky analysis for the sum of data of the first 6.5 years of the COMPTEL mission.

\begin{tabular}{lcccc}
\hline$\gamma$-ray Source & $1-3 \mathrm{MeV}$ & $3-10 \mathrm{MeV}$ & $10-30 \mathrm{MeV}$ & Source Type \\
\hline Crab & $\sim 110$ & $\sim 76$ & $\sim 44$ & pulsar + nebula \\
3C 273 & 11.5 & 10.8 & 6.2 & FSRQ \\
3C 279 & 5.3 & 5.7 & 4.9 & FSRQ \\
PKS 0528+134 & $-b$ & 3.0 & 6.0 & FSRQ \\
PKS 1622-297 & $<3$ & $<3$ & 4.1 & FSRQ \\
3C 454.3 & $<3$ & 5.3 & $<3$ & FSRQ \\
Cen A & 5.7 & 5.3 & 3.0 & Radio Galaxy \\
\hline
\end{tabular}

a Flat-Spectrum Radio Quasar

${ }^{\mathrm{b}}$ Unknown due to uncertainties in removing the strong Crab signal

occuring at $\mathrm{MeV}$-energies. However, we emphasize that for final conclusions a dedicated source analysis has to be carried out.

\section{SUMMARY}

We have applied the maximum-likelihood method to COMPTEL all-sky data of different time periods. By simultaneously fitting models for the different diffuse emission components, this analysis method provided quantitative all-sky results significances and fluxes - on point sources. An all-sky summary on their timeaveraged fluxes and significances is thereby provided. After the Crab - pulsar plus nebula - the quasar $3 \mathrm{C} 273$ was found to be the most significant COMPTEL $\mathrm{MeV}$-source, having time-averaged fluxes of the order of $10 \%$ to $15 \%$ of the Crab. Additional evidence for previously unknown source features has been found as well.

ACKNOWLEDGMENTS: The COMPTEL project is supported by the German government through DARA grant 50 QV 9096 8, by NASA under contract NAS5-26645, and by the Netherlands Organisation for Scientific Research (NWO).

\section{REFERENCES}

1. Schönfelder, V., et al., ApJS 86, 657 (1993).

2. Strong, A.W., et al., Proc. 2nd INTEGRAL Workshop, ESA SP-382, 533 (1997).

3. Bloemen, H., et al., Proc. 3rd INTEGRAL Workshop, in press (1999).

4. Blom, J.J., et al., COMPTEL High-Latitude Gamma-Ray Sources (Ph.D. Thesis), ISBN 90-9010945-5, (1997).

5. Bloemen, H., et al., these proceedings.

6. Bloemen, H., et al., ApJS 92, 419 (1994).

7. Schönfelder, V., et al., ApJS submitted (1999). 